\title{
One-Day Acceptance and Commitment Therapy Compared to Support for Depressed Migraine Patients: a Randomized Clinical Trial
}

\author{
Lilian N. Dindo ${ }^{1,2} \cdot$ Ana Recober $^{3} \cdot$ Chadi A. Calarge ${ }^{2,4} \cdot$ Bridget M. Zimmerman $^{5} \cdot$ Aliza Weinrib $^{6}$. \\ James N. Marchman ${ }^{7} \cdot$ Carolyn Turvey $^{8}$
}

Published online: 20 December 2019

(C) The American Society for Experimental NeuroTherapeutics, Inc. 2019

\begin{abstract}
In patients with migraine, depression is associated with poorer medical prognosis, decreased quality of life, and increased risk of suicidality and disability; yet, behavioral interventions have rarely been investigated. The current study compared the efficacy of two 1-day (5- to 6-h) interventions for co-occurring migraine and depression: (1) acceptance and commitment therapy plus migraine education (ACT-ED), and (2) support plus migraine education (SED). One hundred and thirty-six patients with comorbid depression and migraine were randomized to a treatment. One hundred and three $(76 \%)$ completed the ACT-ED $(N=56)$ or S-ED $(N=47)$ workshop. Primary outcomes were depression diagnosis and symptoms. Secondary outcomes were anxiety symptoms, headache-related disability and general functioning, and quality of life. Assessments were completed at baseline and 3 and 6 months following the workshop. At the 6-month follow-up, on categorical outcomes, a significantly greater number of people in the ACTED condition no longer met criteria for a major depressive episode and exhibited a $\geq 50 \%$ drop in symptoms on the Hamilton Rating Scale of Depression. Similarly, though, weaker results were found when examining depressive symptoms dimensionally. On secondary outcomes, people in the ACT-ED condition exhibited significantly greater improvements in anxiety, headache-related disability, and quality of social relationships, compared to S-ED, No differences between groups were observed in general functioning. A 1-day (5- to 6-h) ACT workshop can deliver substantial and lasting benefits to depressed migraineurs, over and above those provided by group support and education. This approach is an attractive alternative to weekly psychotherapy. Clinicaltrials.gov \# NCT02108678
\end{abstract}

Key Words Acceptance and commitment therapy $(\mathrm{ACT}) \cdot$ Illness management $\cdot$ Migraine $\cdot$ Depression

Electronic supplementary material The online version of this article (https://doi.org/10.1007/s13311-019-00818-0) contains supplementary material, which is available to authorized users.

Lilian N. Dindo

lilian.dindo@bcm.edu

1 Houston VA HSR\&D Center for Innovations in Quality, Effectiveness and Safety, Michael E. DeBakey Veterans Affairs Medical Center, Holcombe, Houston, TX 77030, USA

2 Department of Internal Medicine, Section of Health Services Research, Baylor College of Medicine, Houston, TX, USA

3 University of Pennsylvania \& the Children's Hospital of Philadelphia, Philadelphia, PA, USA
4 Department of Pediatrics, Baylor College of Medicine, Houston, TX, USA

5 Department of Biostatistics, University of Iowa, Iowa City, IA, USA

6 Department of Anesthesia and Pain Management, Toronto General Hospital, University Health Network, Toronto, Ontario, Canada

Department of Psychology, University of Iowa, Iowa City, IA, USA

8 Department of Psychiatry, University of Iowa, Iowa City, IA, USA 


\section{Introduction}

Migraine is a chronic disorder that affects approximately 38 million US residents [1]. It is characterized by episodic, severe headache pain and functional impairment. The direct medical costs of migraine are estimated at 17 billion dollars annually [2], and productivity losses add another 13 billion dollars annually [1]. In addition to the pain and associated disability, migraine patients are at an elevated risk for psychiatric disorders [3]. Depression, in particular, is three times more common among migraineurs than in the general population; and this rate is even higher in migraine patients presenting to clinical settings [1-3]. This comorbidity is of major public health significance as it results in decreased quality of life, poorer response to migraine and depression treatment, and overall worse medical prognosis as well as increased risk for suicidality, medication overuse and disability $\left[{ }^{3}\right]$. Importantly, the economic burden of migraine doubles when there is depression [4]. Yet, despite its deleterious impact, depression in migraineurs is underdiagnosed and undertreated [6]. Anxiety disorders are also associated with migraine [7]. Migraine patients have a two-fold increased risk of an anxiety disorder diagnosis, highlighting the high comorbidity of depression and anxiety disorders with migraines [8]. Notably, effective therapeutic strategies directly targeting depression and anxiety in migraineurs are lacking.

Acceptance and commitment therapy (ACT) is a behavioral intervention with established efficacy in the treatment of chronic pain, depression, and anxiety and with growing evidence of efficacy in a range of other psychiatric and medical disorders [9]. Importantly, when presented as a brief intervention, ACT has resulted in positive outcomes [10, 11]. For example, in a small, uncontrolled pilot trial, patients with co-occurring depression and migraine were assigned to a 1-day ACT plus Education (ACT-ED) group workshop or to Treatment as Usual (TAU). Promising results were found with $77 \%$ of the participants in the ACT-ED arm remitting from depression compared to only $8 \%$ of those in TAU [12]. The intervention, based on ACT, targeted behavioral avoidance and promoted engagement in values-based living, processes implicated in depression and disability in chronic pain patients, including migraineurs [13]. The innovative "1-day workshop" format offered the potential for optimal treatment adherence and treatment completion, the lack of which is often the greatest obstacle to effective delivery of mental health services [14]. Presenting the treatment as a "workshop" was also better suited for individuals who may not be explicitly seeking specialized mental health care [15].

Building on this promising work, we conducted a randomized controlled trial contrasting the ACT-ED intervention to an active support plus migraine education (S-ED) workshop, in a larger group of patients with co-occurring major depressive disorder and migraine. The S-ED condition was intended to control for treatment elements that pose rival explanations for the effectiveness of the ACT-ED condition, including nonspecific treatment elements such as therapist attention, expectation for improvement, and group support, as well as benefits of migraine education. This design allowed us to compare the efficacy of these two interventions and to assess whether there was an important additive effect of ACT training above the supportive and educational group environment. We hypothesized that, at the 6-month follow-up, patients in the ACT-ED workshop would exhibit a significantly greater decline in symptoms of depression and anxiety and improvements in functioning, compared to S-ED.

\section{Methods}

\section{Participants}

Participants were recruited from hospitals, including headache clinics and primary care clinics, and surrounding communities, via referrals, mass email, brochures, and posted flyers. Brochures and flyers stated: "If you have migraines and are having a hard time adjusting to your illness, consider participating in a research study....If you are eligible you would be invited to attend a 1-day workshop focused on ways to manage migraine and life stressors." Those interested in the study completed an online web screening or a phone screening.

The screening inventory was completed by 4880 individuals, ages $18-70$, with a history of migraine. Of those, 501 individuals met the following screening cutoffs: (1) obtained a score of 2 or more on the ID Migraine, a widely used 3-item screening tool with high positive predictive value for the presence of migraines [16]; (2) reported 4-12 migraine days over the previous month; (3) scored 10 or greater on the Patient Health Questionnaire-8 (PHQ-8) [17]; (4) no history of brain injury; (5) if currently using psychotropic or headache medications, use of these medications must have begun at last 4 weeks before the intake interview; (6) no history of schizophrenia, bipolar affective disorder or current substance abuse.

Of these, 177 people (35\%) completed an in-person assessment, consisting of a diagnostic interview and self-report questionnaires. The remaining people who screened positive could not be reached $(n=281)$ or did not come in for an inperson assessment $(n=43)$. The eligibility criteria for the treatment phase included (1) a diagnosis of current major depressive episode on the Structured Clinical Interview for Diagnostic and Statistical Manual for Mental Disorders Fourth Edition (SCID-IV; DSM-IV [18, 19]; (2) a score of 17 or greater on the Hamilton Rating Scale for Depression (HRSD) [20]; (3) a medical chart diagnosis of migraine; and (4) no imminent suicidality requiring urgent clinical attention. Following the in-person assessment, 41 patients were excluded (Fig. 1). Eligible participants $(N=136)$ were randomly 


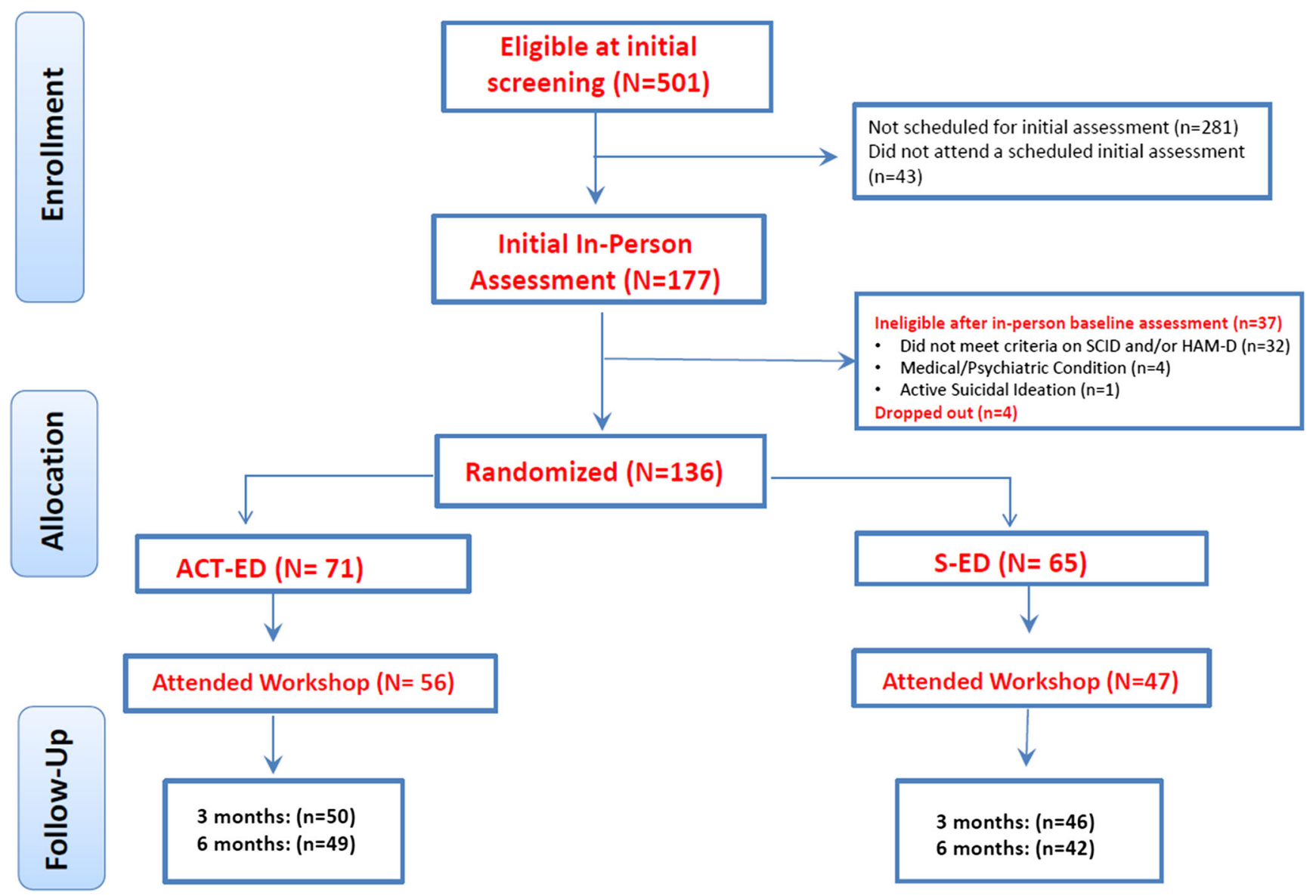

Fig. 1 CONSORT trial flow diagram

assigned to the ACT-ED $(n=71)$ or S-ED $(n=65)$ workshop. Of those, 56 attended the ACT-ED workshop; and 47 attended the S-ED workshop. Thus, $24 \%$ of those randomized to a workshop did not attend. All participants who came to the workshop completed it $(100 \%$ completion of treatment protocol).

This study was fully approved by an Institutional Review Board, and all participants provided written informed consent. The study is also registered in clinicaltrials.gov (NCT02108678).

\section{Design}

Participants were assessed prior to the workshop and again 3 and 6 months postintervention. Assessors were blind to treatment condition. Eligible participants were randomly assigned to ACT-ED or S-ED, using the method of permuted blocks.

\section{Treatments}

The ACT-ED workshops lasted 5-6 h, included 4-8 patients, and involved training in ACT and education about migraine. The ACT component of the workshop emphasized approaches to managing troubling thoughts, feelings and pain sensations (e.g., learning how to recognize and develop cognitive distance from unhelpful thoughts, such as "I can't take this pain anymore," and learning how to willingly face experiences that cannot be changed), while promoting effective and committed actions to achieve life goals. The education component $(1 \mathrm{~h})$, developed by a headache specialist (A.R.), involved educating participants about triggers and prodromes, risk factors for migraine chronification, effective use of medical treatments, and psychological and lifestyle factors known to contribute to migraine. Each ACT-ED workshop was implemented by two psychologists. The clinical content of the ACT workshop has been described in pragmatic detail by Dindo and colleagues [21]. A list of exercises completed during the workshop can also be found in Supplemental Document 1.

The S-ED workshop also lasted about 5-6 h, included 4-8 patients, and was led by two psychologists. The same educational topics listed above about migraine were presented, and participants discussed issues and experiences related to the topic. Additionally, diaphragmatic breathing was taught and practiced; and a passive progressive relaxation exercise (approximately $30 \mathrm{~min}$ ) was completed.

The manuals for the ACT-ED and S-ED workshops are available upon request. 


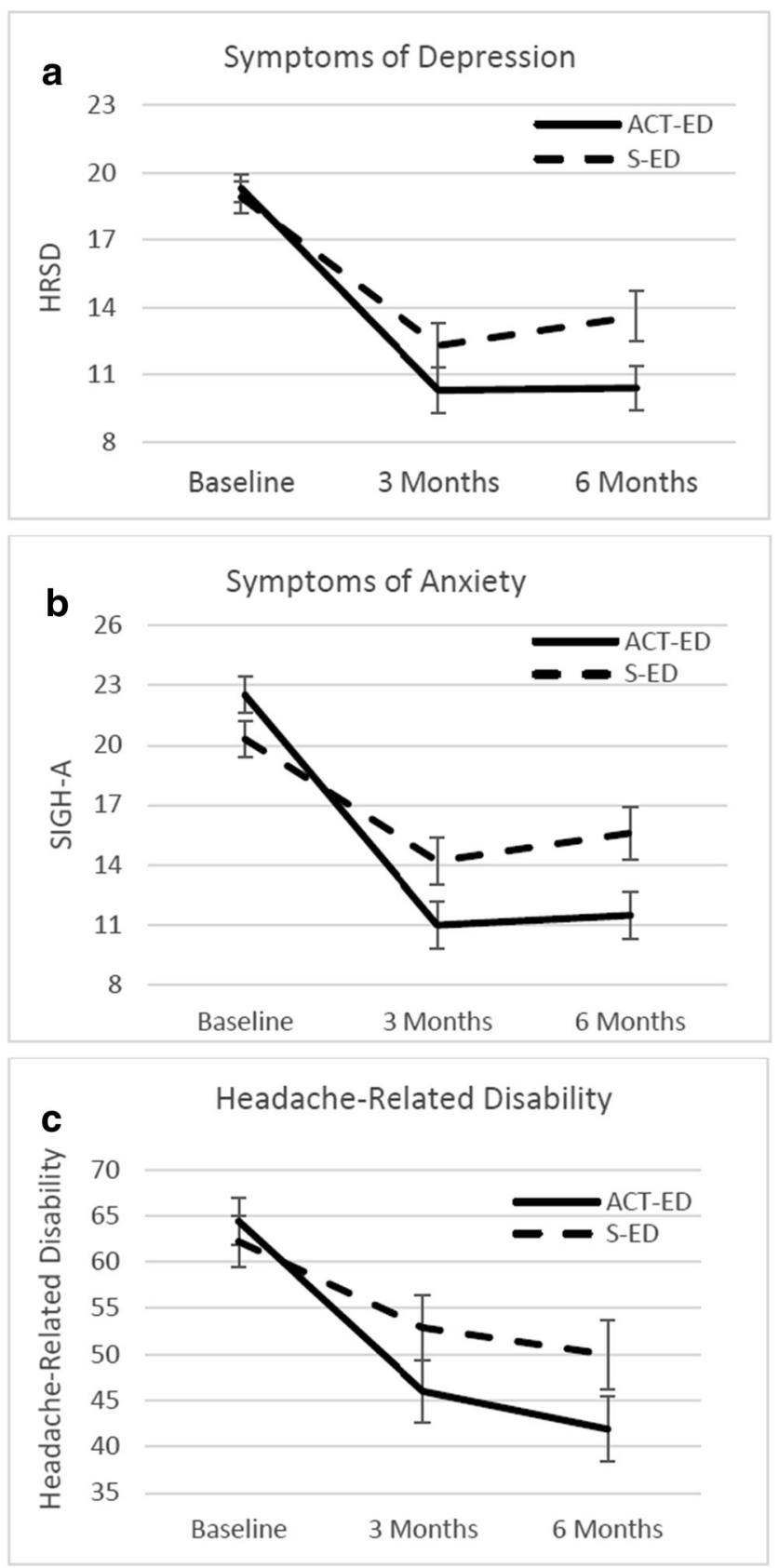

Fig. 2 Changes in depression symptoms, anxiety symptoms, and headache disability for 6 months following an ACT-ED or S-ED workshop

\section{Outcome Measures}

\section{Primary Outcome: Depression}

The main a priori outcome measures for this study were the 17-item HRSD [ 20] and the depression module of the SCIDIV [ 18,19$]$. Response rates were assessed by examining the proportion of participants showing $50 \%$ or greater decline on the HRSD the proportion of participants who no longer met depression criteria on SCID-IV. Symptom-level changes in depression were assessed by examining the HRSD total score.

\section{Secondary Outcomes}

Several secondary outcomes were assessed. The Structured Interview Guide for the Hamilton Anxiety Rating Scale (SIGH-A) was used to assess self-reported anxiety symptoms [22]. Headache-specific functioning was assessed with the Headache Disability Inventory (HDI) [23], which assesses the burden of headaches by inquiring about perceived impact of headaches on daily activities and functioning. Higher scores indicate greater impairment. General functioning was assessed with the 36-item World Health Organization Disability Assessment Schedule 2.0 (WHO-DAS 2.0) [24]. Lower scores indicate greater functioning. Quality of life was assessed with the World Health Organization Quality of Life (WHO-QOL). It assesses physical health, psychological well-being, social relationships and environment (e.g., satisfaction with living place and access to health care) [25].

\section{Data Analyses}

Demographic and clinical variables that describe study participant characteristics were compared between the two intervention groups. The two-sample $t$ test was used to compare continuous variables, and Pearson's $\chi 2$ test was used to compare categorical variables.

Generalized linear mixed model for binary data was used to assess the impact of treatment condition on SCID-IV diagnosis of depression, treatment response defined as $>50 \%$ reduction on HRSD or SIGH-A [26], and a reduction of $\geq 29$ points on the HDI [23]. Treatment effect was estimated as odds ratio (OR) with $95 \%$ confidence interval and as number needed to treat (NNT).

Linear mixed-model analysis for repeated measures was used to compare the effect of treatment status on depressive symptom severity (HRSD), anxiety symptom severity (SIGHA), headache-related disability (HDI), general functioning (WHO-DAS) and quality-of-life domains (WHO-QOL). The fixed effects in the linear mixed model were treatment status (ACT-ED versus S-ED), time (baseline, 3 months, and 6 months), and treatment-by-time interaction effect. Of interest in assessing the efficacy of ACT-ED over S-ED is to show a significant treatment-time interaction effect, which indicates differences in mean change from baseline over the two followup times in outcome measures between treatment groups. In addition, the linear mixed-model analysis also included performing tests of mean contrast to examine specific comparisons of interest, such as change over time within each treatment group and between-group comparison of means and mean changes at each time point. The estimate of treatment effect was presented as the difference in mean change 
from baseline to 3 months and 6 months between treatment groups (with $95 \%$ confidence interval), and also as Cohen's $d$ effect size.

All hypothesis tests were two-tailed, with Bonferroni adjustment applied to the $p$ value (and confidence intervals) to account for treatment group comparisons assessed at two time points. Additionally, in testing for significance of within treatment group change from baseline, Bonferroni adjusted $p$ value accounted for 4 tests, i.e., within-group change for 2 groups at 2 time points ( $4 \times$ unadjusted $p$ value). The $p$ values reported in the tables for the marginal tests (within treatment group change and comparison of mean change between treatment groups at each time point) are the Bonferroni adjusted $\mathrm{p}$ values.

A $p$ value $<0.05$ was considered as statistically significant. All statistical analyses were performed using procedures from SAS version 9.4 (SAS/STAT 14.3, SAS Software, Inc., Carey, NC). Of note, linear mixed model for repeated measures using SAS Proc MIXED includes all data points available, not just those with both 3 and 6 months data. In the presence of missing data, under the assumption of missing at random (MAR), linear mixed model analysis which can handle incompletely observed subjects and uses a likelihood estimation method (i.e., SAS MIXED procedure) provides correct likelihoods and leads to valid estimates.

\section{Results}

\section{Patient Characteristics}

Table 1 presents demographic and clinical characteristics of study participants. Participants were mostly white, relatively well educated, and mostly female, consistent with the higher rates of both depression and migraine in women [7]. Almost all participants were taking acute medications (prescribed or over the counter) for their migraine-related pain. In contrast, preventive migraine medications were being used by only a minority, also consistent with global data [27]. Approximately one-third of the participants in each group were also taking an antidepressant. There were no differences between participants in the ACT-ED and S-ED conditions on these variables (Table 1).

\section{Primary Outcome (Depression)}

Using the a priori definition of response for HRSD of $\geq 50 \%$ reduction in symptoms, a significantly greater proportion of participants treated with ACT-ED responded to treatment compared to S-ED over the two follow-up times [overall treatment effect $\mathrm{F}(1,97)=8.39 ; p=0.005]$. At 3 months, $62.8 \%$ in ACT-ED compared to $35.3 \%$ in S-ED responded to treatment $(\mathrm{OR}=3.10 ; \mathrm{NNT}=3.6 ; p=0.021)$. At 6 months $51.4 \%$ in ACT-ED versus $28.2 \%$ in S-ED exhibited an HRSD response $(\mathrm{OR}=2.70, \mathrm{NNT}=3.9 ; p=0.059)$ (Table 2).

On the SCID-IV, $100 \%$ of participants met diagnostic criteria for a current major depressive episode (MDE) At the 3- and 6-months follow-up, there were significantly fewer participants with current major depressive episode in the ACT-ED group than in the S-ED group [overall treatment effect $\mathrm{F}(1,97)=4.44 ; p=0.038]$. At 3 months, 26.9 in ACT$\mathrm{ED}$ and $40.7 \mathrm{in} \mathrm{S}-\mathrm{ED}$ had current $\mathrm{MDE}(\mathrm{OR}=0.54, p=0.32)$. At 6 months, $22.6 \%$ in ACT-ED and $45.8 \%$ in S-ED group had current $\mathrm{MDE}(\mathrm{OR}=0.35, p=0.044)$ (Table 2).

Analyses of symptoms on the HRSD suggested a trend (nonsignificant) towards a treatment-by-time interaction effect, $\mathrm{F}(2,109)=2.51, p=0.086$. Assessment of this interaction, planned a priori, showed a significant mean decrease in severity of depressive symptoms in both ACT-ED and ED-S groups at 3 months and 6 months. The difference in mean change observed between treatment groups at 3 months was not significant $(p=0.21)$, though the effect size was moderate $(0.34)$. At 6 months a greater mean decrease was seen in the ACT-ED
Table 1 Demographic Variables

\begin{tabular}{llll}
\hline Variable & $\begin{array}{l}\text { ACT-ED } \\
(n=56)\end{array}$ & $\begin{array}{l}\text { S-ED } \\
(n=47)\end{array}$ & $p$ value \\
\hline Age, years (mean/SD) & $36.9(14.9)$ & $34.4(12.6)$ & 0.36 \\
Female sex, $n(\%)$ & $47(84 \%)$ & $38(81 \%)$ & 0.68 \\
Caucasian race, $n(\%)$ & $42(75 \%)$ & $36(77 \%)$ & 0.85 \\
$>12$ years of education, n (\%) & $32(59 \%)$ & $27(57 \%)$ & 0.67 \\
Employed or in School, $n(\%)$ & $44(82 \%)$ & $39(83 \%)$ & 0.42 \\
Age of onset of migraines, years (mean/SD) & $18.0(10.2)$ & $20.2(11.1)$ & 0.39 \\
Taking antidepressant, $n(\%)$ & $17(32 \%)$ & $15(33 \%)$ & 0.95 \\
Number of migraine/headache days during & $7(3.2)$ & $7.9(3.5)$ & 0.40 \\
$\quad$ month prior to baseline visit (mean/SD) & $48(86 \%)$ & $42(89 \%)$ & 0.53 \\
Taking abortive anti-migraine medication, $n(\%)$ & $19(34 \%)$ & $17(37 \%)$ & 0.50 \\
Taking preventive anti-migraine medication, $n(\%)$ & & \\
\hline
\end{tabular}

ACT-ED = Acceptance and Commitment Training plus Migraine Education; S-ED = Support plus Education 
Table 2 Estimate of the mean proportion of the binary response outcome for HRSD, SCID-IV, SIGH-A, and HDI at 3 and 6 months follow-up from generalized linear mixed model analysis to compare the effect of ACTED versus S-ED

\begin{tabular}{|c|c|c|c|c|c|}
\hline & \multirow{2}{*}{$\begin{array}{l}\text { ACT-ED }(n=56) \\
\text { Mean Proportion }(95 \% \\
\text { CI) }\end{array}$} & \multirow{2}{*}{$\begin{array}{l}\text { ED-S }(n=47) \\
\text { Mean Proportion }(95 \% \\
\text { CI) }\end{array}$} & \multicolumn{3}{|l|}{ ACT-ED vs. ED-S } \\
\hline & & & $\begin{array}{l}\text { Odds Ratio (95\% } \\
\text { CI) }\end{array}$ & NNT & $\begin{array}{l}p \\
\text { value }\end{array}$ \\
\hline \multicolumn{6}{|c|}{ HRSD $>50 \%$ decline } \\
\hline 3 months & $62.8 \%(48.3,75.4)$ & $35.3 \%(22.6,50.5)$ & $3.10(1.15,8.35)$ & 3.6 & 0.021 \\
\hline 6 month & $51.4 \%(37.4,65.2)$ & $28.2 \%(16.5,43.8)$ & $2.70(0.97,7.49)$ & 3.9 & 0.059 \\
\hline \multicolumn{5}{|c|}{ Treatment effect: $F(1,97)=8.39$} & 0.005 \\
\hline \multicolumn{6}{|c|}{ SCID-IV depression } \\
\hline 3 months & $26.9 \%(16.2,41.1)$ & $40.7 \%(27.3,55.7)$ & $0.54(0.20,1.47)$ & 7.2 & 0.326 \\
\hline 6 month & $22.6 \%(13.0,36.4)$ & $45.8 \%(31.6,60.7)$ & $0.35(0.12,0.98)$ & 4.3 & 0.044 \\
\hline \multicolumn{5}{|c|}{ Treatment effect: $F(1,97)=4.44$} & 0.038 \\
\hline \multicolumn{6}{|c|}{ SIGH-A $>50 \%$ decline } \\
\hline 3 months & $63.7 \%(48.0,77.0)$ & $41.8 \%(27.1,58.2)$ & $2.45(0.85,7.05)$ & 4.6 & 0.113 \\
\hline 6 month & $51.8 \%(36.3,67.0)$ & $20.2 \%(10.0,36.6)$ & $4.25(1.28,14.03)$ & 3.2 & 0.014 \\
\hline \multicolumn{5}{|c|}{ Treatment effect: $F(1,80)=7.85$} & 0.006 \\
\hline \multicolumn{6}{|c|}{ HDI $>29$ decline } \\
\hline 3 months & $36.8 \%(24.4,51.3)$ & $11.5 \%(4.8,25.4)$ & $4.47(1.23,16.26)$ & 4.0 & 0.019 \\
\hline 6 month & $37.7 \%(25.2,52.0)$ & $12.1 \%(4.9,26.7)$ & $4.40(1.20,16.18)$ & 3.9 & 0.022 \\
\hline \multicolumn{5}{|c|}{ Treatment effect: $F(1,96)=10.26$} & 0.002 \\
\hline
\end{tabular}

NNT $=$ Number needed to treat; ACT-ED = Acceptance and Commitment Training plus Migraine Education; $\mathrm{S}$ ED = Support plus Education; HRSD = Hamilton Rating Scale for Depression; SCID-IV = Structured Clinical Interview for DSM-IV: SIGH-A = Structured Interview Guide for the Hamilton Anxiety Rating Scale; HDI = Headache Disability Inventory group $(-8.9 ; 95 \% \mathrm{CI}-1.7,-6.2)$ compared to the S-ED group $(-5.3 ; 95 \% \mathrm{CI}-8.3,-2.3)$. The mean decrease in HRSD at the 6-month follow-up was $3.6(95 \% \mathrm{CI}-0.1,7.4)$ more in ACTED than in ED-S (effect size $=0.46 ; p=0.055$; Table 3 and Fig. 2).

\section{Secondary Outcomes}

\section{Anxiety}

Evaluation of response to treatment on the SIGH-A, defined a priori as $\geq 50 \%$ reduction in symptoms, showed a significant difference overall between the two treatment groups $[\mathrm{F}(1,80)=7.85 ; p=0.006]$. At 3 months, $63.7 \%$ in ACT-ED compared to $41.4 \%$ in $\mathrm{S}-\mathrm{ED}$ responded to treatment, $(\mathrm{OR}=2.45 ; \mathrm{NNT}=4.6 ; p=0.11)$. At 6 months, $51.8 \%$ in ACT-ED versus $20.2 \%$ in ED-S responded to treatment $(\mathrm{OR}=4.25 ; \mathrm{NNT}=3.2 ; p=0.01$; Table 2). Participants in the ACT-ED condition showed significantly greater improvement over the two follow-up times in anxiety symptom severity compared to those in ED-S [treatment-by-time interaction: $\mathrm{F}(2,113)=7.10, p \leq 0.001]$. Assessment of this interaction effect showed a significant mean decrease in SIGH-A in both ACT-ED and S-ED groups at 3 months and 6 months, with a significantly greater decrease in the ACT-ED group than in the S-ED group at both follow-ups (Table 3 and Fig. 2).

\section{Headache-Related Disability}

Analysis of the HDI suggested a trend towards a treatment-bytime interaction effect $[\mathrm{F}(2,92)=2.92, p=0.059]$. There was a significant mean decrease in HDI score in both ACT-ED and ED-S groups at 3 months and 6 months. The mean decrease in HDI score at the 3-month follow-up was 9.1 (95\% CI 0.5 , 19.0) more in the ACT-ED than in the ED-S group (effect size $=0.44 ; p=0.067$; Table 4). The mean decrease in HDI score at the 6-month follow-up was 10.3 (95\% CI: 0.2, 20.4) more in the ACT-ED than in the ED-S group (effect size $=0.48 ; p=0.043$; Table 4 and Fig. 2).

Using the a priori definition of $a \geq 29$ decline in HDI total score, a significantly greater proportion of participants treated with ACT-ED exhibited a decline compared to ED-S over the two follow-up times [overall treatment effect $\mathrm{F}(1,96)=10.26 ; p=0.002]$. The proportion that showed $\mathrm{HDI} \geq 29$ decline was $36.8 \%$ in ACT-ED group and $11.5 \%$ in ED-S at 3 months $(\mathrm{OR}=4.47 ; p=0.02)$. At 6 months, it was $37.7 \%$ in ACT-ED versus $12.1 \%$ in ED-S $(\mathrm{OR}=4.40 ; p=0.02)$ (Table 2).

\section{WHO-QOL}

Significant differences between ACT-ED and ED-S in mean score change over time were observed for WHOQOL psychological well-being [treatment-by-time 
Table 3 Estimate of mean and mean change from baseline at 3 and 6 months follow-up from linear mixed model analysis to compare the effect of ACTED versus S-ED on HRSD and SIGH-A

\begin{tabular}{|c|c|c|c|c|c|c|c|}
\hline & \multicolumn{2}{|c|}{ ACT-ED $(n=56)$} & \multicolumn{2}{|c|}{$\mathrm{ED}-\mathrm{S}(n=47)$} & \multicolumn{3}{|l|}{ ACT-ED vs. ED-S } \\
\hline & $\begin{array}{l}\text { Mean } \\
\text { (SE) }\end{array}$ & $\begin{array}{l}\text { Mean change } \\
(95 \% \mathrm{CI})\end{array}$ & $\begin{array}{l}\text { Mean } \\
\text { (SE) }\end{array}$ & $\begin{array}{l}\text { Mean change } \\
(95 \% \text { CI })\end{array}$ & $\begin{array}{l}\text { Difference mean } \\
\text { change }(95 \% \mathrm{CI})\end{array}$ & Effect size & $p$ value \\
\hline \multicolumn{8}{|l|}{ HRSD } \\
\hline Baseline & $19.3(0.6)$ & - & $18.9(0.7)$ & - & & & \\
\hline 3 months & $10.3(1.0)$ & $-9.0^{\wedge}(-11.7,-6.4)$ & $12.3(1.0)$ & $-6.5^{\wedge}(-9.3,-3.7)$ & $-2.5(-6.1,1.0)$ & 0.34 & 0.205 \\
\hline 6 month & $10.4(1.0)$ & $-8.9^{\wedge}(-11.7,-6.2)$ & $13.6(1.1)$ & $-5.3^{\wedge}(-8.3,-2.3)$ & $-3.6(-7.4,0.1)$ & 0.46 & 0.055 \\
\hline \multicolumn{7}{|c|}{ Treatment $*$ Time Interaction: $F(2,109)=2.51$} & 0.086 \\
\hline \multicolumn{8}{|c|}{$\underline{\text { SIGH-A }}$} \\
\hline Baseline & $22.5(0.9)$ & - & $20.3(0.9)$ & - & & & \\
\hline 3 months & $11.0(1.2)$ & $-11.4^{\wedge}(-14.1,-8.7)$ & $14.2(1.2)$ & $-6.1^{\wedge}(-8.9,-3.2)$ & $-5.4(-8.9,-1.8)$ & 0.73 & 0.002 \\
\hline 6 month & $11.5(1.2)$ & $-10.9^{\wedge}(-14.0,-7.8)$ & $15.6(1.3)$ & $-4.7 * *(-8.0,-1.4)$ & $-6.2(-10.3,-2.1)$ & 0.74 & 0.001 \\
\hline \multicolumn{7}{|c|}{ Treatment $*$ Time Interaction: $F(2,113)=7.10$} & 0.001 \\
\hline
\end{tabular}

ACT-ED = Acceptance and Commitment Training plus Migraine Education; S-ED = Support plus Education; HRSD = Hamilton Rating Scale for Depression; SIGH-A = Structured Interview Guide for the Hamilton Anxiety Rating Scale

${ }^{\wedge}$ Significant change from baseline Bonferroni adjusted $p<0.001$

**Significant change from baseline Bonferroni adjusted $p<0.01$

Interaction: $\mathrm{F}(2,143)=3.07 ; p=0.050]$ and social relationships [treatment-by-time Interaction: $\mathrm{F}(2,178)=4.61$; $p=0.011]$. A trend was observed for WHO-QOL environment [treatment-by-time Interaction: $\mathrm{F}(2,180)=2.73 ; p=$ 0.068] (see Table 4). For psychological well-being, the ACT-ED group exhibited greater improvements than the ED-S group at both follow-up times (3.1 vs. $1.4 ; p=$ 0.056 at 3 months; 3.6 vs. $1.7 ; p=0.068$ at 6 months). Social relationships showed greater improvement in ACTED versus ED-S at 3 months (1.1 vs. $-0.3 ; p=0.006)$ but not at 6 months ( 1.5 vs. $0.4 ; p=0.128)$. The same result was seen for environment with larger increase in ACT-ED versus $\mathrm{ED}-\mathrm{S}$ at 3 months (1.9 vs. $-0.1 ; p=0.045)$ but with no significant difference at 6 months $(2.2$ vs. $1.0 ; p=$ $0.535)$. WHO-QOL physical health significantly increased over the two follow-up times in both groups, with no significant difference in mean score change between the two groups [treatment-by-time Interaction: $F(2,182)=$ $0.96 ; p=0.385]$.

\section{WHO-DAS}

Mean WHO-DAS score decreased over time in both the ACTED and ED-S groups, with no significant difference in the magnitude of change over time between the two groups [treatment-by-time interaction $\mathrm{F}(2,1.82)=1.28 ; p=0.282]$. At the 6-month follow-up, the improvement in the disability score was $9.6(95 \%$ CI $4.3,14.9)$ for ACT-ED and $6.1(95 \%$ CI $0.3,6.1$ ) for ED-S (see Table 4).

\section{Moderator Analyses}

Gender, race, education, income, and medication use were examined as possible effect moderators of ACT-ED and SED. No significant effects were observed.

Of note, all the aforementioned analyses were also run on the full intent-to-treat sample; including all study participants who were randomized. Results of these intent-to-treat analyses are presented in supplemental Tables 1-3 (paralleling Tables 2, 3, and 4). Overall, the results of these analyses were very similar to the "completer" analyses (those who attended a workshop).

\section{Discussion}

This randomized controlled trial was designed to move us towards identifying an effective and efficient intervention for depression (as well as anxiety) and disability in patients with migraine. To that end, this study directly addressed the question of whether the previously demonstrated efficacy of group ACT workshops for depressed migraineurs is primarily due to nonspecific factors such as group support, therapist attention and education and to query whether ACT intervention provides added value over and above those nonspecific factors for this population. Therefore, 1-day ACT-ED and S-ED interventions were compared in a group of patients with comorbid depression and migraine with 3-and 6-month follow-up. 
Table 4 Estimate of mean and mean change from baseline at 3 and 6 months follow-up from linear mixed model analysis to compare the effect of ACTED versus S-ED on Functional Outcomes

\begin{tabular}{|c|c|c|c|c|c|c|c|}
\hline & \multicolumn{2}{|c|}{ ACT-ED $(n=56)$} & \multicolumn{2}{|c|}{$\mathrm{ED}-\mathrm{S}(n=47)$} & \multicolumn{3}{|l|}{ ACT-ED vs. ED-S } \\
\hline & $\begin{array}{l}\text { Mean } \\
\text { (SE) }\end{array}$ & $\begin{array}{l}\text { Mean change } \\
(95 \% \text { CI })\end{array}$ & $\begin{array}{l}\text { Mean } \\
\text { (SE) }\end{array}$ & $\begin{array}{l}\text { Mean change } \\
(95 \% \text { CI })\end{array}$ & $\begin{array}{l}\text { Diff mean change } \\
(95 \% \mathrm{CI})\end{array}$ & Effect size & $p$ value \\
\hline \multicolumn{8}{|c|}{ HDI Total Score } \\
\hline Baseline & $64.4(2.5)$ & - & $62.2(2.8)$ & - & & & \\
\hline 3 months & $46.0(3.4)$ & $-18.4^{\wedge}(-25.7,-11.3)$ & $52.9(3.6)$ & $-9.3 *(-17.1,-1.4)$ & $-9.1(-19.0,0.5)$ & 0.44 & 0.067 \\
\hline 6 month & $41.9(3.5)$ & $-22.5^{\wedge}(-29.9,-15.1)$ & $50.0(3.8)$ & $-12.2 * *(-20.3,-4.0)$ & $-10.3(-20.4,-0.2)$ & 0.48 & 0.043 \\
\hline \multicolumn{7}{|c|}{ Treatment $*$ Time Interaction: $F(2,92)=2.92$} & 0.059 \\
\hline \multicolumn{8}{|c|}{ WHO-QOL Psychological well-being } \\
\hline Baseline & $17.4(0.5)$ & - & $17.6(0.5)$ & - & & & \\
\hline 3 months & $20.5(0.6)$ & $3.1^{\wedge}(1.8,4.3)$ & $19.0(0.7)$ & $1.4 *(0.1,2.8)$ & $1.7(0.0,3.3)$ & 0.46 & 0.056 \\
\hline 6 month & $21.0(0.6)$ & $3.6^{\wedge}(2.1,5.0)$ & $19.3(0.6)$ & $1.7 *(0.1,3.3)$ & $1.9(-0.1,3.9)$ & 0.44 & 0.068 \\
\hline \multicolumn{7}{|c|}{ Treatment $*$ Time Interaction: $F(2,143)=3.07$} & 0.050 \\
\hline \multicolumn{8}{|c|}{ WHO-QOL Social relationships } \\
\hline Baseline & $9.4(0.3)$ & - & $9.6(0.4)$ & - & & & \\
\hline 3 months & $10.5(0.3)$ & $1.1 * *(0.4,2.0)$ & $9.3(0.4)$ & $-0.3(-1.1,0.6)$ & $1.4(0.4,2.5)$ & 0.62 & 0.006 \\
\hline 6 month & $10.9(0.3)$ & $1.5^{\wedge}(0.6,2.6)$ & $10.0(0.4)$ & $0.4(-0.6,1.5)$ & $1.1(-0.2,2.4)$ & 0.38 & 0.128 \\
\hline \multicolumn{7}{|c|}{ Treatment $*$ Time Interaction: $F(2,178)=4.61$} & 0.011 \\
\hline \multicolumn{8}{|c|}{ WHO-QOL Environment } \\
\hline Baseline & $28.4(0.6)$ & - & $29.2(0.7)$ & - & & & \\
\hline 3 months & $30.3(0.7)$ & $1.9 * *(0.4,3.3)$ & $29.1(0.7)$ & $-0.1(-1.7,1.4)$ & $2.0(0.0,3.9)$ & 0.47 & 0.045 \\
\hline 6 month & $30.6(0.7)$ & $2.2 * *(0.4,4.1)$ & $30.2(0.7)$ & $1.0(-1.0,3.0)$ & $1.2(-1.2,3.7)$ & 0.23 & 0.535 \\
\hline \multicolumn{7}{|c|}{ Treatment $*$ Time Interaction: $F(2,180)=2.73$} & 0.068 \\
\hline \multicolumn{8}{|c|}{ WHO-QOL Physical health } \\
\hline Baseline & $21.5(0.6)$ & - & $21.0(0.7)$ & - & & & \\
\hline 3 months & $25.1(0.7)$ & $3.6^{\wedge}(2.2,5.1)$ & $23.5(0.7)$ & $2.5^{\wedge}(1.0,4.1)$ & $1.1(-0.8,3.0)$ & 0.27 & 0.395 \\
\hline 6 month & $25.5(0.7)$ & $4.0^{\wedge}(2.2,5.8)$ & $23.7(0.7)$ & $2.7 * *(0.8,4.7)$ & $1.3(-1.2,3.7)$ & 0.24 & 0.477 \\
\hline \multicolumn{7}{|c|}{ Treatment $*$ Time Interaction: $F(2,182)=0.96$} & 0.385 \\
\hline \multicolumn{8}{|c|}{ WHO-DAS Total } \\
\hline Baseline & $31.1(2.0)$ & - & $29.0(2.2)$ & - & & & \\
\hline 3 months & $23.2(2.1)$ & $-7.9^{\wedge}(-12.1,-3.8)$ & $24.9(2.2)$ & $-4.1(-8.5,0.4)$ & $-3.9(-9.4,1.6)$ & 0.33 & 0.227 \\
\hline 6 month & $21.5(2.1)$ & $-9.6^{\wedge}(-14.9,-4.3)$ & $22.9(2.3)$ & $-6.1 *(-11.9,-0.3)$ & $-3.5(-10.6,3.6)$ & 0.23 & 0.537 \\
\hline \multicolumn{7}{|c|}{ Treatment $*$ Time Interaction: $F(2,182)=1.28$} & 0.282 \\
\hline
\end{tabular}

ACT-ED = Acceptance and Commitment Training plus Migraine Education; S-ED = Support plus Education; HDI = Headache Disability

${ }^{\wedge}$ Significant change from baseline Bonferroni adjusted $p<0.001$

**Significant change from baseline Bonferroni adjusted $p<0.01$

*Significant change from baseline Bonferroni adjusted $p<0.05$

This study showed that the ACT-ED intervention resulted in significant improvements in depression, based on the proportion of participants who responded to treatment (exhibited a drop of $50 \%$ or more on HRSD) and the proportion of participants who no longer met DSM-IV criteria for MDE. Although nonsignificant, a trend was observed with the ACT-ED group showing more improvements on depressive symptom severity (HSRD) than SED. It is not uncommon for categorical and dimensional outcomes of depression to yield different results [28]. However, in the current study, the effect sizes on the dimensional outcomes were moderate to large. Thus, the overall message is similar across the categorical and dimensional measures of depression: ACT has a moderate advantage over S-ED.

The ACT-ED intervention also resulted in significantly greater improvements in anxiety symptoms. In terms of functioning and quality of life, significantly greater 
improvements were observed in headache-related disability, psychological well-being, and quality of social relationships for the ACT-ED group compared to the S-ED group. No significant group differences were observed in general functioning.

In both the current investigation and the original pilot investigation, individuals with co-occurring depression and migraine who participated in the ACT workshop achieved a > $70 \%$ rate of remission for depression. In the previous trial, the ACT intervention was compared to treatment as usual; whereas here we have compared the total ACT workshop package (which includes ACT content, therapist attention, group dynamics and migraine education) against a strong comparateur (which includes therapist attention, group dynamics, migraine education and opportunities for self-reflection but omits the ACT content). This replicates the previous finding regarding depression remission rates after one-time ACT intervention in this population, while extending previous research by suggesting that the addition of ACT coping strategies has important effects on distress and headache disability. This is particularly notable, given the known difficulty in finding advantages for psychological treatments over active control treatments [29]. At the same time, with a $54 \%$ remission rate of depression at 6 months for patients who participated in the support workshop, these findings highlight the power of a one-time group format in providing re-moralization to patients living with a difficult and isolating illness. Group therapy has been thought to provide a sense of universality [30] and may enhance a sense of common humanity, a foundation of self-compassion [31]. Further, educational interventions have been found to improve health outcomes for patients with migraine [32]. The strong finding of depression remission for the S-ED condition suggests that group support and education constitute a powerful intervention in themselves.

Weekly psychotherapy appointments have been a staple of behavioral intervention for generations. Since the advent of cognitive-behavioral therapy, depression has been thought to require 12-16 sessions of individual treatment. With new research highlighting an opportunity to provide briefer treatments, the question is naturally raised: are one-time interventions as helpful as ongoing sessions for people living with chronic and re-current conditions, such as migraine and depression? The effect sizes obtained in this study are comparable to those reported by a series of meta-analyses for weekly cognitive-behavioral therapy and other psychotherapeutic treatments for depression [33]. In addition, the effect sizes are generally higher than those obtained for pharmacological treatment of mild-to-moderate depression [34], without the risks of side-effects and the challenges of weaning. Furthermore, similar to our findings, a recent meta-analysis also showed that psychotherapeutic interventions for depression can have positive impacts on symptoms of anxiety [35]. This study adds to a growing body of literature showing that brief psychotherapeutic interventions can be highly effective, transdiagnostically impactful and acceptable to medical patients [9]. For example, 1-day ACT interventions have shown promise in patients with diabetes, obesity, multiple sclerosis, and cardiovascular disease risk factors [11]. Notably, to the best of our knowledge, this is the first study to compare a 1day ACT intervention to an active treatment condition in a population with comorbid psychiatric and medical problems, thus taking an important step forward for this burgeoning literature.

In addition to its efficacy, the ACT intervention featured in this line of research has several key pragmatic strengths. First, for patients with functional limitations and practical barriers such as transportation difficulties or family and work responsibilities [36], a 1-day workshop ensures treatment adherence and completion. A meta-analysis of 125 studies on outpatient psychotherapy found that $50 \%$ of patients drop out of treatment prematurely [37], and nearly $40 \%$ terminate treatment after the first or second visit [13]. Such high dropout levels are problematic because patients continue to experience symptoms and, thus, may increase healthcare utilization in other ways. Overall, the commonly encountered difficulties with treatment adherence and completion in psychotherapy were not an obstacle with the workshop format [38]. Once we got participants to the workshop, we were able to retain them for the whole treatment ( $100 \%$ treatment completion rate). At the same time, a sizable number of participants $(24 \%)$ were eligible for a workshop but did not attend one. A greater understanding of the reasons for "no-shows" or what can be done for distressed patients who do not present for therapy is needed. It may be that interventions that can reach patients at home, such as web- or mobile-based applications, may be more effective at targeting patients who do not present to clinic. Similar to how we offer a "workshop" rather than "therapy," which is less stigmatizing, e-health programs likely should also be presented in a manner that is acceptable to patients who are distressed but not explicitly seeking mental health care [39]. In the current context, it is also possible that patients did not want to attend a group intervention. Research has shown that potential clients sometimes express reservations about group psychotherapy, including fear of criticism, rejection, or the expectation to self-disclose [40].

The current study raises important questions about how best to assess improvements in functioning and quality of life, the sensitivity of generic measures of disability as compared to disease-specific measures of disability, and the value of identifying endpoints in trials that reflect the patient's desired outcomes. This study found greater improvements for the ACT group in terms of the proportion of patients who showed improvements in headache-specific disability in comparison to the support group. In contrast, there were no differences between groups in general functioning. A generic measure of disability, such as the WHO-DAS may be too nonspecific to 
capture changes that may result from an ACT workshop; although specific subscales such as well-being and quality of social relationships (aspects of quality of life) improved significantly more in ACT-ED than in S-ED. This finding aligns with a key therapeutic target of ACT interventions - helping patients clarify their values and engage in actions consistent with those values, and much of this work naturally centers around important relationships. A large amount of literature has shown that prioritizing meaningful relationships is linked with better mental and physical health outcomes [41]. Qualitative research may help to better identify what types of in behavior occur due to values-based interventions like ACT, whether these outcomes are meaningful to participants, and how they can be measured using standardized questionnaires.changes

\section{Limitations and Future Directions}

Consistent with previous ACT research, positive effects on distress and disability were maintained or strengthened at the final 6-month follow-up period [42]. During the workshop, participants are taught new ways to approach their thoughts and emotions and are given opportunities to practice behavioral skills in session. As participants continue to practice these new skills after the workshop, they may develop more flexible patterns of behavior, which positively impact wellbeing on an ongoing basis. Our clinical vision is to develop a one-time intervention with long-lasting impact; therefore, the longevity of the treatment effect must be empirically investigated. Future studies should examine whether the positive treatment effects obtained persist over longer periods of time than this study's 6-month follow-up, or whether they begin to wear off at certain times. This could provide important information about timing for booster sessions. Future studies should examine the effect of an ACT-ED intervention on migraine-related pain, medication use and healthcare utilization. Given the bidirectional influence of depression and migraine, an improvement in one disorder should result in improvement in the other.

\section{Conclusion}

Depression and/or anxiety in patients with migraine is of major public health significance because these mental health problems are common and result in greater disability and overall worse prognosis than migraine alone [43, 44]. Despite the high prevalence and deleterious impact, mental health problems are often underdiagnosed and undertreated in patients with migraine, due to poor recognition of distress, lack of evidence to guide interventions [5] and a dearth of behavioral treatments that are appealing to a population that does not see itself as needing mental health care. This represents a missed opportunity, as both depression and anxiety are modifiable problems [45]; and psychological interventions have a long history of success in managing patients suffering from both mental health and medical conditions [46]. This study suggests that a 1-day workshop featuring ACT strategies, group support and migraine education offers a promising and efficient way forward for this patient population. It also highlights the noteworthy impact of group support and education in providing re-moralization, as well as the additional benefit can be attained from learning ACT.

Acknowledgments This work was made possible by grant number K23MH097827 from the National Institute of Mental Health awarded to Lilian N. Dindo and was partially supported by the use and resources of the Houston VA HSR\&D Center for Innovations in Quality, Effectiveness and Safety (CIN13-413). The opinions expressed reflect those of the authors and not necessarily those of the Department of Veterans Affairs, the US government, or Baylor College of Medicine.

Required Author Forms Disclosure forms provided by the authors are available with the online version of this article.

\section{Compliance with Ethical Standards}

The authors declare that that they have no conflicts of interest.

\section{References}

1. Bigal ME, Lipton RB. The epidemiology, burden, and comorbidities of migraine. Neurol Clin 2009;27:321-334.

2. Lipton RB, Stewart WF, Diamond S, Diamond ML, Reed M. Prevalence and burden of migraine in the United States: Data from the American Migraine Study II. Headache 2001;41:646-657.

3. Jette N, Patten S, Williams J, Becker W, Wiebe S. Comorbidity of migraine and psychiatric disorders-A national population-based study. Headache 2008;48:501-516.

4. Lake AE, 3rd, Rains JC, Penzien DB, Lipchik GL. Headache and psychiatric comorbidity: Historical context, clinical implications, and research relevance. Headache 2005;45:493-506.

5. Pesa J, Lage MJ. The medical costs of migraine and comorbid anxiety and depression. Headache 2004;44:562-570.

6. Buse DC, Rupnow MF, Lipton RB. Assessing and managing all aspects of migraine: Migraine attacks, migraine-related functional impairment, common comorbidities, and quality of life. Mayo Clin Proc 2009;84:422-435.

7. Hamelsky SW, Lipton RB. Psychiatric comorbidity of migraine. Headache 2006;46:1327-1333.

8. McWilliams LA, Goodwin RD, Cox BJ. Depression and anxiety associated with three pain conditions: Results from a nationally representative sample. Pain 2004;111:77-83.

9. Dimidjian S, Arch JJ, Schneider RL, Desormeau P, Felder JN, Segal ZV. Considering meta-analysis, meaning, and metaphor: A systematic review and critical examination of "third wave" cognitive and behavioral therapies. Behav Ther 2016;47:886-905.

10. Dindo L, Van Liew JR, Arch JJ. Acceptance and commitment therapy: A transdiagnostic behavioral intervention for mental health and medical conditions. Neurotherapeutics 2017;14:546-553.

11. Dindo L. One-day acceptance and commitment training workshops in medical populations. Curr Opin Psychol 2015;2:38-42. 
12. Dindo L, Recober A, Marchman JN, Turvey C, O'Hara MW. Oneday behavioral treatment for patients with comorbid depression and migraine: A pilot study. Behav Res Ther 2012;50:537-543.

13. McCracken LM, Vowles KE. Acceptance of chronic pain. Curr Pain Headache Rep 2006;10:90-94.

14. Wierzbicki M, Pekarik, G. The relationship between expected and actual psychotherapy treatment duration. Psychotherapy 1986;23: 532-534.

15. Mohr DC, Hart SL, Howard I, et al. Barriers to psychotherapy among depressed and nondepressed primary care patients. Ann Behav Med 2006;32:254-258.

16. Lipton RB, Dodick D, Sadovsky R, et al. A self-administered screener for migraine in primary care: The ID Migraine Validation Study. Neurology 2003;61:375-382.

17. Kroenke K, Spitzer RL, Williams JB. The PHQ-9: Validity of a brief depression severity measure. J Gen Intern Med 2001;16: 606-613.

18. Diagnostic and Statistical Manual of Mental Disorders, Text Revision. 4th ed. Washington, DC: American Psychiatric Association; 2000.

19. Spitzer RL, Williams JB, Gibbon M, First MB. The Structured Clinical Interview for DSM-III-R (SCID). I: History, rationale, and description. Arch Gen Psychiatry 1992;49:624-629.

20. Williams JB. A structured interview guide for the Hamilton Depression Rating Scale. Arch Gen Psychiatry 1988;45:742-747.

21. Dindo L, Weinrib A, Marchman J. One-day ACT workshops for patients with chronic health problems and associated emotional disorders. In: Levin ME, Twohig MP, Krafft J, eds. Innovations in ACT. CA: New Harbinger; 2019.

22. Shear MK, Vander Bilt J, Rucci P, et al. Reliability and validity of a structured interview guide for the Hamilton Anxiety Rating Scale (SIGH-A). Depress Anxiety 2001;13:166-178.

23. Jacobson GP, Ramadan NM, Aggarwal SK, Newman CW. The Henry Ford Hospital Headache Disability Inventory (HDI). Neurology 1994;44:837-842.

24. Rehm J, Bedirhan Ustun T, Saxena S, et al. On the development and psychometric testing of the WHO screening instrument to assess disablement in the general population. Int J Methods Psychiatr Res 1999;8:110-122.

25. Skevington SM, Lotfy M, O'Connell KA. The World Health Organization's WHOQOL-BREF quality of life assessment: Psychometric properties and results of the international field trial. A report from the WHOQOL group. Qual Life Res 2004;13:299310.

26. O'Hara MW, Stuart S, Gorman LL, Wenzel A. Efficacy of interpersonal psychotherapy for postpartum depression. Arch Gen Psychiatry 2000;57:1039-1045.

27. Blumenfeld AM, Bloudek LM, Becker WJ, et al. Patterns of use and reasons for discontinuation of prophylactic medications for episodic migraine and chronic migraine: Results from the Second International Burden of Migraine Study (IBMS-II). Headache 2013;53:644-655.

28. Kessler RC. The categorical versus dimensional assessment controversy in the sociology of mental illness. J Health Soc Behav 2002;43(2):171-188.
29. Budd R, Hughes I. The Dodo Bird Verdict-controversial, inevitable and important: a commentary on 30 years of meta-analyses. Clin Psychol Psychother 2009;16(6):510-522.

30. Yalom I, Leszcz M. The Theory and Practice of Group Psychotherapy. 5th. New York: IUP; 2005.

31. Neff KD. The Role of Self-Compassion in Development: A Healthier Way to Relate to Oneself. Hum Dev 2009;52(4):211214.

32. Smith TR, Nicholson RA, Banks JW. Migraine education improves quality of life in a primary care setting. Headache 2010;50(4):600 612.

33. Cuijpers P, van Straten A, Andersson G, van Oppen P. Psychotherapy for depression in adults: a meta-analysis of comparative outcome studies. J Consult Clin Psychol 2008;76(6):909922.

34. Fournier JC, DeRubeis RJ, Hollon SD, et al. Antidepressant drug effects and depression severity: a patient-level meta-analysis. Jama 2010;303(1):47-53.

35. Weitz E, Kleiboer A, van Straten A, Cuijpers P. The effects of psychotherapy for depression on anxiety symptoms: a meta-analysis. Psychol Med 2018;48(13):2140-2152.

36. Spleen AM, Lengerich EJ, Camacho FT, Vanderpool RC. Health care avoidance among rural populations: results from a nationally representative survey. J Rural Health 2014;30(1):79-88.

37. Wierzbicki M, Pekarik, G. A Meta-Analysis of Psychotherapy Dropout. Professional Psychology: Research and Practice. 1993.

38. Najavits LM. The problem of dropout from "gold standard" PTSD therapies. F1000prime Rep 2015;7:43.

39. Cummings NA, Cummings JL, Johnson JN, Baker NJ. Behavioral health in primary care: a guide for clinical integration. Madison: Psychosocial Press; 1997.

40. Zipora Shechtman \& Abeer Kiezel. Why Do People Prefer Individual Therapy Over Group Therapy?, Int J Group Psychother 2016; 66:4, 571-591.

41. Holt-Lunstad J. Why social relationships are important for physical health: A systems approach to understanding and modifying risk and protection. Annu Rev Psychol 2018;69:437-458.

42. Luoma JB, Kohlenberg BS, Hayes SC, Fletcher L. Slow and steady wins the race: A randomized clinical trial of acceptance and commitment therapy targeting shame in substance use disorders. J Consult Clin Psychol 2012;80:43-53.

43. Buse DC, Andrasik F. Behavioral medicine for migraine. Neurol Clin 2009;27:445-465.

44. Bigal ME, Lipton RB. Modifiable risk factors for migraine progression. Headache 2006;46:1334-1343.

45. Barlow DH. Psychological treatments. Am Psychol 2004;59:869878.

46. Barlow DH, Bullis JR, Comer JS, Ametaj AA. Evidence-based psychological treatments: An update and a way forward. Annu Rev Clin Psychol 2013;9:1-27.

Publisher's Note Springer Nature remains neutral with regard to jurisdictional claims in published maps and institutional affiliations. 